\title{
Solidification microstructure of centrifugally cast Inconel 625
}

\author{
*Silvia Barella', Andrea Gruttadauria', Carlo Mapelli', Davide Mombelli', Paolo Taiana', Matteo Bosatra², and \\ Alberto Morini ${ }^{2}$ \\ 1. Dipartimento di Meccanica, Politecnico di Milano, via La Masa 1, 20156 Milano, Italy \\ 2. Fondinox, via Marconi 42/48, 26010 Sergnano (CR), Italy
}

\begin{abstract}
Centrifugal casting is a foundry process allowing the production of near net-shaped axially symmetrical components. The present study focuses on the microstructural characterization of centrifugally cast alloys featuring different chemical compositions for the construction of spheres applied in valves made of alloy IN625 for operation at high pressure. Control of the solidification microstructure is needed to assure the reliability of the castings. Actually, a Ni-base superalloy such as this one should have an outstanding combination of mechanical properties, high temperature stability and corrosion resistance. Alloys such as IN625 are characterised by a large amount of alloying elements and a wide solidification range, so they can be affected by micro-porosity defects, related to the shrinkage difference between the matrix and the secondary reinforcing phases (Nb-rich carbides and Laves phase). In this study, the microstructure characterization was performed as a function of the applied heat treatments and it was coupled with a calorimetric analysis in order to understand the mechanism ruling the formation of micro-porosities that can assure alloy soundness. The obtained results show that the presence of micro-porosities is governed by morphology and by the size of the secondary phases, and the presence of the observed secondary phases is detrimental to corrosion resistance.
\end{abstract}

Key words: alloy IN625; centrifugal casting; solidification defects; Nb carbides; Laves phases; micro-porosity; corrosion
CLC numbers: TG146. $1^{+} 5$
Document code: A
Article ID: 1672-6421(2017)04-304-09

$\mathrm{C}$ Yentrifugal casting is a foundry technique mainly used to produce axial symmetrical products, because it exploits the centrifugal force created during solidification of the material, which is cast in a rotating round shape that is die cooled to promote cast alloy solidification $^{[1]}$. The number of metallurgical defects in components obtained through this process is less than in other foundry processes, because the nonmetallic inclusions and the entrapped gas are removed as solidification progresses. Moreover, the macrostructure of the product is mainly columnar due to heat extraction

\footnotetext{
*Silvia Barella

Ph.D., her research activities focus on solidification processes (casting and welding), hot and cold plastic deformation and the heat treatment of iron and aluminum based alloy. She also deals with the metallographic and mechanical characterization and phase transformations as a result of thermal and mechanical treatments. She is the secretariat of the Steelmaking Committee of the Italian Association of Metallurgy. She is the author of about 50 peer reviewed scientific papers.

E-mail: silvia.barella@polimi.it
}

Received: 2017-02-15; Accepted: 2017-05-05 towards the external surface, while it is finely equiaxed in the inner part as a result of the low thermal gradient and the constitutional undercooling caused by segregation in the last solidified volume of the casting ${ }^{[1,2]}$.

Superalloys are characterised by an optimal combination of mechanical properties, creep resistance and corrosion resistance in various environments. They can be employed at homologous $\left(T / T_{\mathrm{m}}\right)$ temperatures that are even higher than $0.5^{[1-4]}$. In particular, for use in a lower temperature range, nickel based superalloys were designed to exploit a solid solution strengthening mechanism ${ }^{[5]}$ in order to also increase corrosion resistance. Although the solution strengthening mechanism is required for use at a low temperature range, the large concentration of the alloying elements coupled with the wide solidification range cause segregation of the chemical elements forming the secondary phase (i.e. $\mathrm{Nb}$ ) in the last solidified fraction of the alloy ${ }^{[6,7]}$. The undesired secondary phases precipitate during the last stages of the solidification process, and they mainly comprise $\mathrm{Nb}$ carbides and the topologically 
close-packed Laves phase. Their formation depends on the ratio between $\mathrm{C}$ and $\mathrm{Nb}$ concentrations featuring the alloy (Fig. 1); note that in intermediate conditions (characterized by the presence of both $\mathrm{Nb}$ carbides and Laves phases) the phase diagram presents a solidification sequence which indicates that $\mathrm{NbCs}$ form before the Laves phase ${ }^{[8-11]}$

The cast products of Ni-based alloys (i.e. IN625) are characterised by a wide solidification range, and this characteristic is usually associated with the presence of micro-porosities.

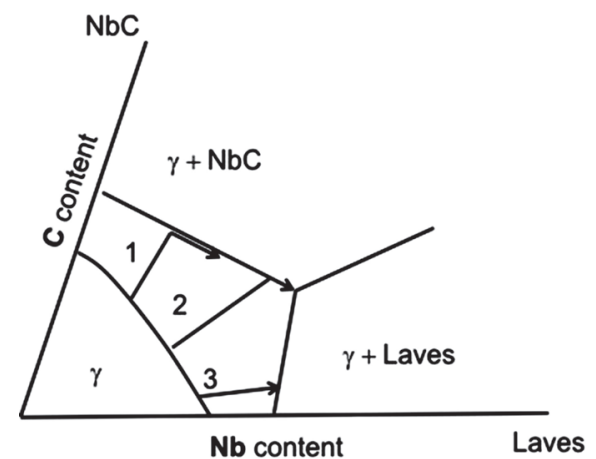

Fig. 1: Pseudo-ternary diagram with solidification sequences for alloy IN625
Such a defect spreads along the thickness and is caused by the shrinkage difference between the matrix and the secondary phases formed in the final stages of solidification ${ }^{[1]}$.

The alloy investigated in the present study was drawn from spheres built for high pressure which were made of alloy IN625 and realized by centrifugal casting.

This study aims at understanding the mechanism ruling the formation of micro-porosity as a function of the morphology and size of the precipitated secondary phases. Moreover, corrosion tests were carried out to find the influence of the microporosities and the secondary phases on the corrosion resistance of the material.

\section{Experimental procedure}

For the present study, four spheres having a $180 \mathrm{~mm}$ diameter were cast. Their chemical compositions (Table 1) were quite similar and within the range characterizing the IN625 alloy.

IN625-2 has a higher Mo concentration than IN625-1. Alloy IN625-3 features a higher concentration of $\mathrm{Nb}$, while the fourth chemical composition is characterized by higher concentrations of $\mathrm{C}, \mathrm{Si}, \mathrm{Nb}$ and $\mathrm{Fe}$ than the other three alloys.

Table 1: Chemical compositions of analysed alloys (wt.\%) and $\mathrm{C} / \mathrm{Nb}$ ratio

\begin{tabular}{cccccccccccc} 
& $\mathbf{C}$ & $\mathrm{Cr}$ & $\mathrm{Ni}$ & $\mathrm{Mn}$ & $\mathrm{Si}$ & $\mathbf{S}$ & $\mathrm{Fe}$ & $\mathrm{Mo}$ & $\mathrm{Nb}$ & $\mathbf{C} / \mathrm{Nb}$ \\
\hline IN625-1 & 0.027 & 21.47 & 63.81 & 0.82 & 0.81 & 0.0018 & 2.16 & 8.19 & 2.28 & 0.0118 \\
IN625-2 & 0.028 & 21.31 & 62.93 & 0.75 & 0.79 & 0.0018 & 2.31 & 9.20 & 2.25 & 0.0124 \\
IN625-3 & 0.028 & 21.25 & 62.03 & 0.81 & 0.78 & 0.002 & 2.65 & 9.15 & 2.85 & 0.009 \\
IN625-4 & 0.059 & 21.13 & 62.60 & 0.69 & 0.86 & 0.003 & 3.25 & 8.11 & 2.92 & 0.021
\end{tabular}

Differential Scanning Calorimetry (DSC), using a LabSys

TG-DTA-DSC 1600 instrument, was carried out on as-cast samples in order to determine the transformation temperatures. Different heating rates of $5,20,30 \mathrm{~K} \cdot \mathrm{min}^{-1}$ were used, from room temperature to the melting point $(1,723 \mathrm{~K})$. Moreover, 5 different cooling rates $\left(40,30,20,10\right.$ and $\left.5 \mathrm{~K} \cdot \mathrm{min}^{-1}\right)$ were used from the molten condition to room temperature. The results were useful for finding the influence of the cooling rates on the solidification sequence.

The as-cast macrostructure along a section of the sphere was revealed using glyceregia etching at $100{ }^{\circ} \mathrm{C}$. Metallographic analyses were performed to characterize the microstructure (at half thickness). Grinded and polished samples were etched by means of a solution composed of $\mathrm{HCl}(35 \mathrm{ml}), \mathrm{CuCl}_{2}$ (1 g), ethanol $(65 \mathrm{ml})$ and $\mathrm{H}_{2} \mathrm{O}_{2}(7$ drops $)$. These analyses were performed using optical microscopy and SEM equipped with EDS and BSE probes. The sample microstructures were observed in the as-cast condition and after two solubilisation heat treatments. These solubilisation treatments were performed at $1,478 \mathrm{~K}$ and $1,548 \mathrm{~K}$, respectively, with a soaking period of $30 \mathrm{~s} \cdot \mathrm{min}^{-1}$ (determined as a function of the specimen thickness). To avoid precipitation, water was used to quench.

The porosity volume fraction was measured by an automatic image analyser.

Finally, corrosion tests were performed on the as-cast and all the heat-treated samples according to the ASTM G4811 standard, to correlate the microstructural changes and the presence of secondary phases and micro-porosities with corrosion resistance. The samples were submerged for 72 hours in a solution of water and $\mathrm{FeCl}_{3}$, and subsequently the corrosion resistance was measured using the weight loss method.

\section{Results}

\subsection{Macrostructural analysis}

Macroetching revealed the centrifugally cast sphere solidification 
structures, with all the analysed samples showing the same macrostructure. An example is shown in Fig. 2.

Three different zones were highlighted in all the analysed samples:

- The chill zone (left border, Fig. 2): the molten metals, in contact with the interior surface of the cooled die, solidified in the form of fine equiaxed grains, finely nucleated due to the intense undercooling;

- The columnar zone: the zone with the widest developed morphology;

- The equiaxed zone: the constitutional supercooling and the

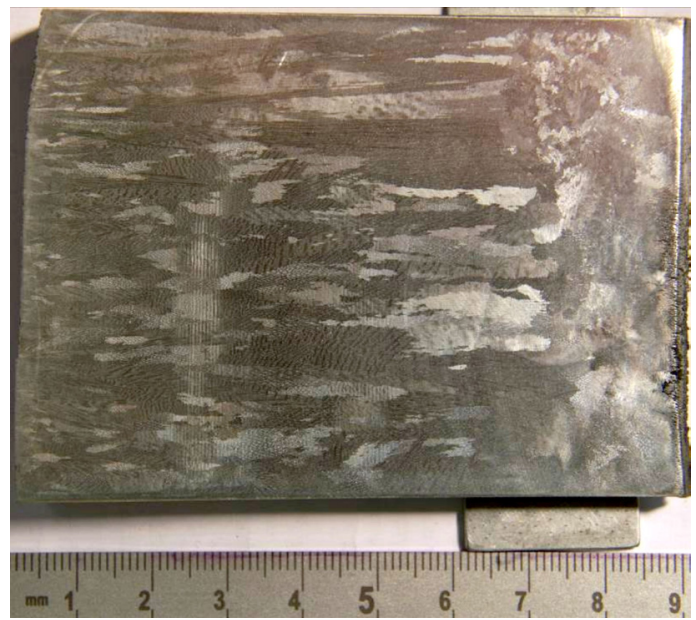

Fig. 2: Macro-etched surface of a sphere section in IN625-3 alloy
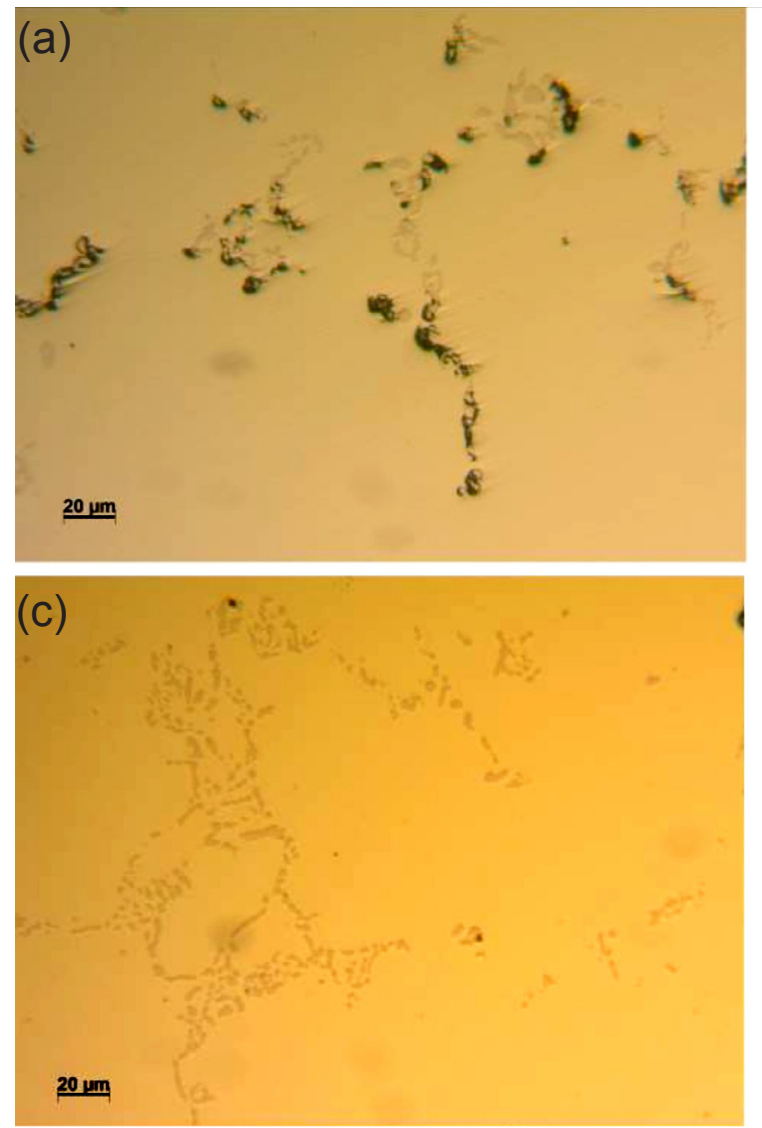

slow growth rate of the solid front create this structure near the interior border of the casting ${ }^{[2,12,13]}$.

\subsection{Microstructural analysis of as-cast samples}

All the as-cast samples, drawn from the columnar zone, presented a grain size corresponding to G-00 (grains average diameter equal to $508 \mu \mathrm{m}$ in accordance to ASTM E112-13 ). Moreover, the microstructural analysis revealed secondary phases and microporosity that surrounded the former.

The micro-porosity volume fraction, detected in the columnar region, is reported in Table 2, where it can be seen that IN625-1 and IN625-2 have a higher amount of these defects than alloys IN625-3 and IN625-4 (Fig. 3).

The secondary phases (Fig. 4) formed during solidification were identified, and their chemical composition was determined using EDX spectroscopy. For all the studied alloys in the as-

Table 2: Volume fraction (\%) of micro-porosities revealed in different analyzed samples

\begin{tabular}{|cc|} 
& Micro-porosity volume fraction (\%) \\
\hline IN625-1 & 0.57 \\
IN625-2 & 0.33 \\
IN625-3 & 0.013 \\
IN625-4 & 0.086 \\
\hline
\end{tabular}
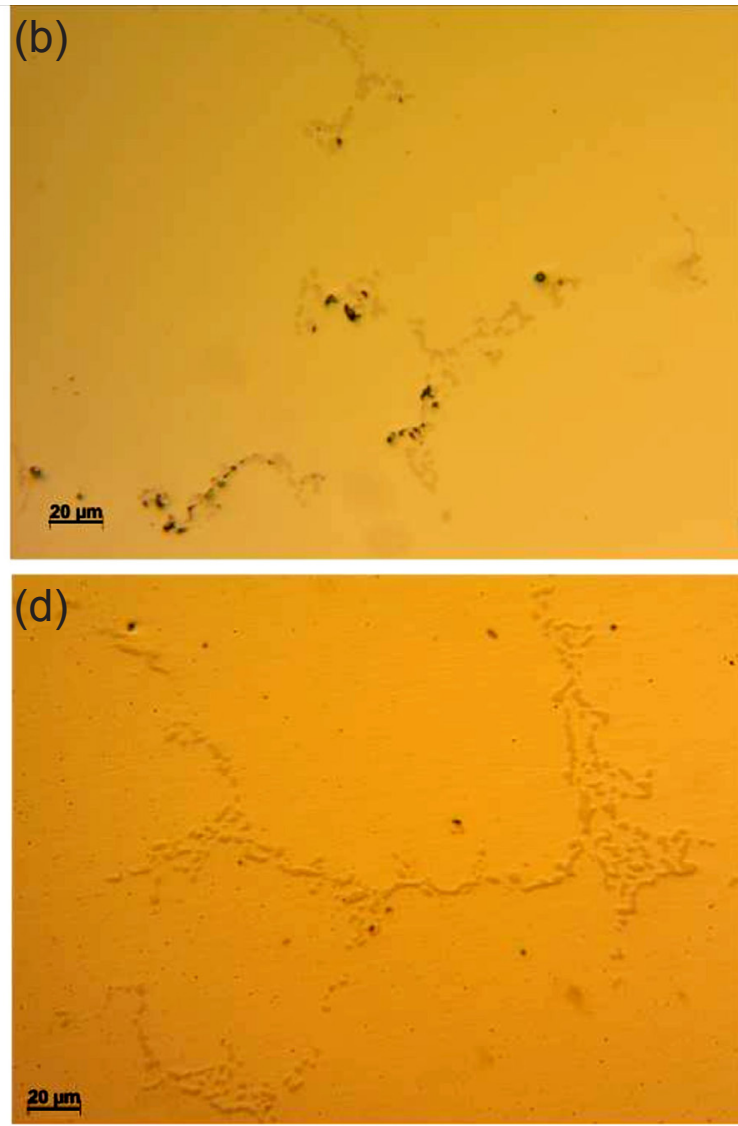

Fig. 3: Micro-porosities and secondary phases observed in alloy: IN625-1 (a), IN625-2 (b), IN625-3 (c) and IN625-4 (d) 
cast condition, the Laves phase was the most frequent secondary phase recognized. To identify the Laves phase, the rates among the alloying elements in atomic percentage (Table 3) was used.

In contrast, niobium carbides $(\mathrm{NbC})$ are almost absent. Only in alloy IN625-4, with the higher C content, were very small particles detected. These probably correspond to $\mathrm{NbC}{ }^{[14]}$ but, due to their small dimension (smaller than the EDS spot), the probe was not able determine their chemical compositions accurately (Fig. 5).

\subsection{Calorimetric analysis}

In all the examined alloys, a similar calorimetric pattern was identified during the heating stage, and two endothermic peaks can be pointed out (Fig. 6). The first peak corresponds to the dissolution of the secondary phases formed during solidification, and the main endothermic peak corresponds to the melting of
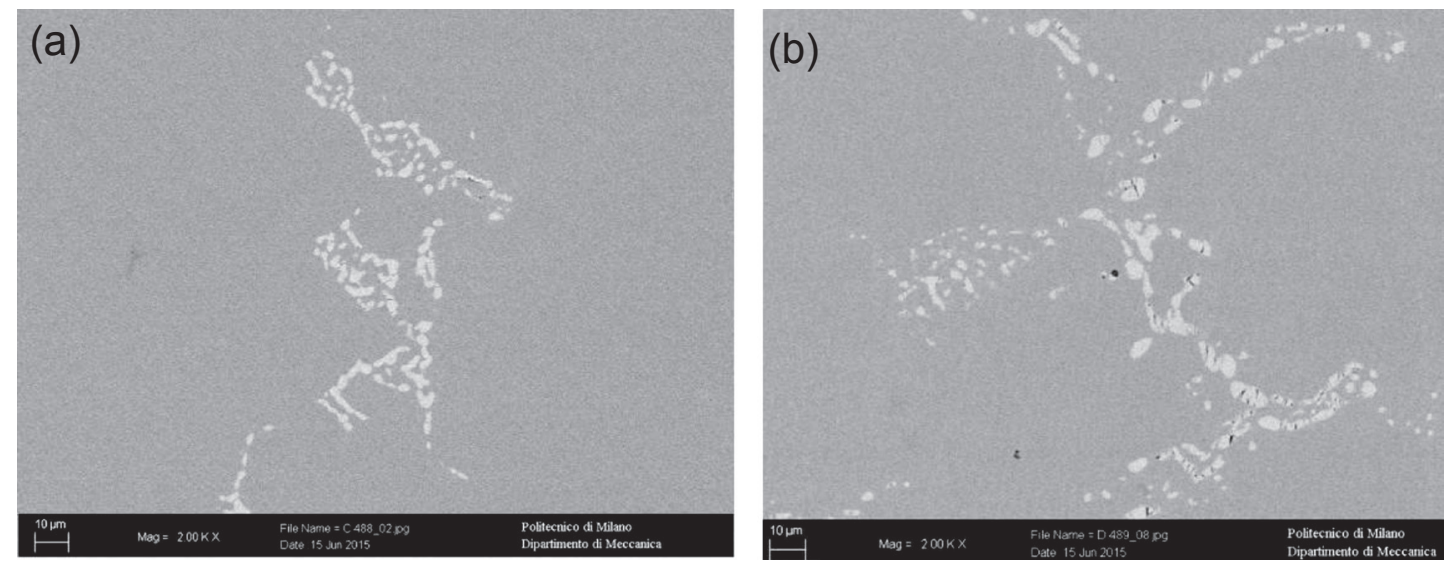

Fig. 4: SEM analysis of samples IN625-3 and IN625-4

Table 3: Average chemical compositions (wt.\%) of Laves phases found in examined alloys

\begin{tabular}{lccccccc} 
& Si & Cr & Fe & Ni & Nb & Mo \\
\hline IN625-1 & 12.14 & 15.28 & 0.61 & 32.51 & 16.24 & 23.22 \\
IN625-2 & 11.19 & 15.67 & 0.74 & 32.35 & 15.11 & 24.94 \\
IN625-3 & 11.40 & 14.26 & 0.81 & 32.47 & 16.66 & 24.40 \\
IN625-4 & 10.46 & 17.64 & 0.96 & 33.06 & 14.12 & 23.76
\end{tabular}

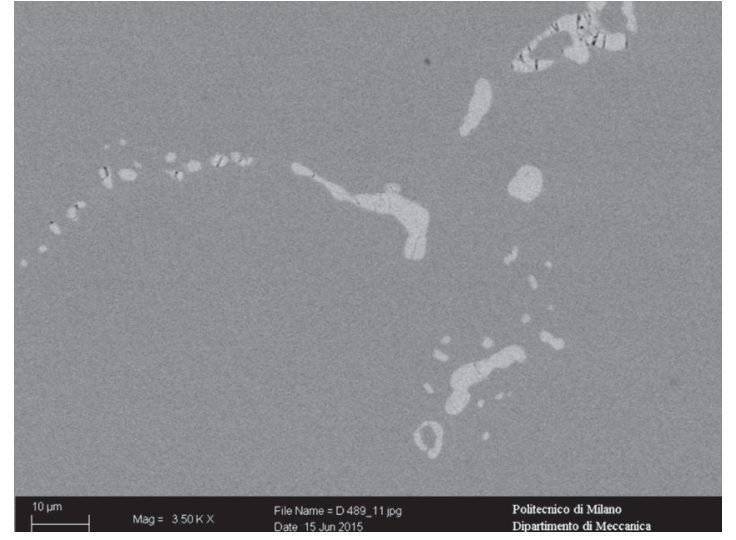

Fig. 5: Detail of NbC precipitation observed in IN625-4

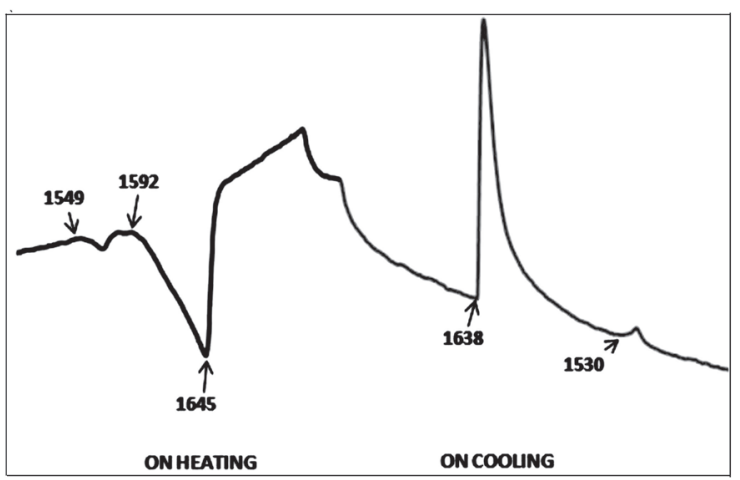

Fig. 6: Heating and cooling stages for alloy IN625-1 at $20 \mathrm{~K} \cdot \mathrm{min}^{-1}$ with indication of transformations and corresponding temperatures the alloy ${ }^{[7]}$. The dissolution temperature of the secondary phases ( $\left.T_{\text {diss }}\right)$ for the four alloys, their liquidus temperatures $\left(T_{\mathrm{L}}\right)$ and the solidus temperatures $\left(T_{\mathrm{S}}\right)$, were determined and collected (Table 4). The values are stated as the average temperatures taken on the different applied scan rates. The applied heating rates and the quite different chemical composition did not imply significant changes of the solidification range for the studied alloys.

The calorimetric pattern obtained during cooling confirms the result of the heating pattern (Fig. 6). For each condition, the liquidus temperature is slightly lower than the same temperature measured at the heating stage, because of the dissipative phenomena involved in the phase transformations. During the cooling stage, a smaller exothermic peak appears at about $1,530 \mathrm{~K}$. Such a peak indicates $\mathrm{NbC}$ formation, while the Laves phases form at a temperature that is at about $100 \mathrm{~K}$ lower ${ }^{[7]}$. Actually, the Laves phase starts its formation after the $\mathrm{NbC}$ 
Table 4: Mean temperatures measured at heating stage (with standard deviations) at different scan rates and calculation of solidification range

\begin{tabular}{ccccc} 
Alloy & $T_{\text {diss }}(\mathbf{K})$ & $T_{\mathbf{S}}(\mathbf{K})$ & $T_{\mathrm{L}}(\mathbf{K})$ & Range $(\mathbf{K})$ \\
\hline IN625-1 & $1546 \pm 5.2$ & $1586 \pm 5.1$ & $1648 \pm 5.2$ & $62 \pm 9.6$ \\
\hline IN625-2 & $1543 \pm 2.5$ & $1584 \pm 9.5$ & $1644 \pm 5.0$ & $60 \pm 7.2$ \\
IN625-3 & $1541 \pm 4.5$ & $1585 \pm 6.7$ & $1639 \pm 4.6$ & $54 \pm 5.8$ \\
IN625-4 & $1545 \pm 5.8$ & $1592 \pm 5.6$ & $1641 \pm 1.7$ & $49 \pm 7.0$
\end{tabular}

precipitation, when the carbon concentration has decreased (Fig. 1). All the analysed alloys show a similar calorimetric pattern but, after the microstructural analysis of the DSC samples, many microstructural differences can be underlined for the different alloys.

In general, the lowest applied cooling rates promote the formation of phases rich in $\mathrm{Nb}$. In alloy IN625-1, characterised by a low $\mathrm{C}$ concentration, two types of $\mathrm{Nb}$-rich precipitates were detected (Fig. 7). The $5 \mathrm{~K} \cdot \mathrm{min}^{-1}$ cooling approximates the equilibrium condition, and the obtained microstructure was characterized by a biphasic constituent featuring $\gamma$-phase nuclei surrounded by $\mathrm{Nb}$ rich granules. At the higher cooling rates, the $\mathrm{Nb}$ precipitates morphology was elongated; this peculiar shape is usually defined as Chinese script ${ }^{[7]}$. Both of these Nb-rich phases morphologies are not associated with micro-porosities, because their surface to volume ratio is higher than those of globular precipitates. For this reason, Chinese scripts are less affected by shrinkage.

The chemical composition of the different $\mathrm{Nb}$-rich precipitates is reported in Table 5. Despite the higher Mo concentration, IN625-2 features the same microstructure. Alloy IN6253 is characterised by a higher $\mathrm{Nb}$ concentration, while the $\mathrm{C}$ concentration is at the same level in the first two alloys. The lowest cooling rates promote $\mathrm{Nb}$-rich phase formation.

In particular, the $5 \mathrm{~K} \cdot \mathrm{min}^{-1}$ scan rate induces the strong precipitation of secondary phases characterised by a Chinese script shape (Fig. 8). In this case, in the same aggregate, two zones with different chemical analysis coexist (Table 6).

Alloy IN625-4 contains the highest concentration of all the alloying elements. There is a high volume fraction of the secondary phases, located at the grain boundaries. Low cooling rates and the high concentration of the alloying elements increase the volume fraction of the secondary phase (Fig. 9).
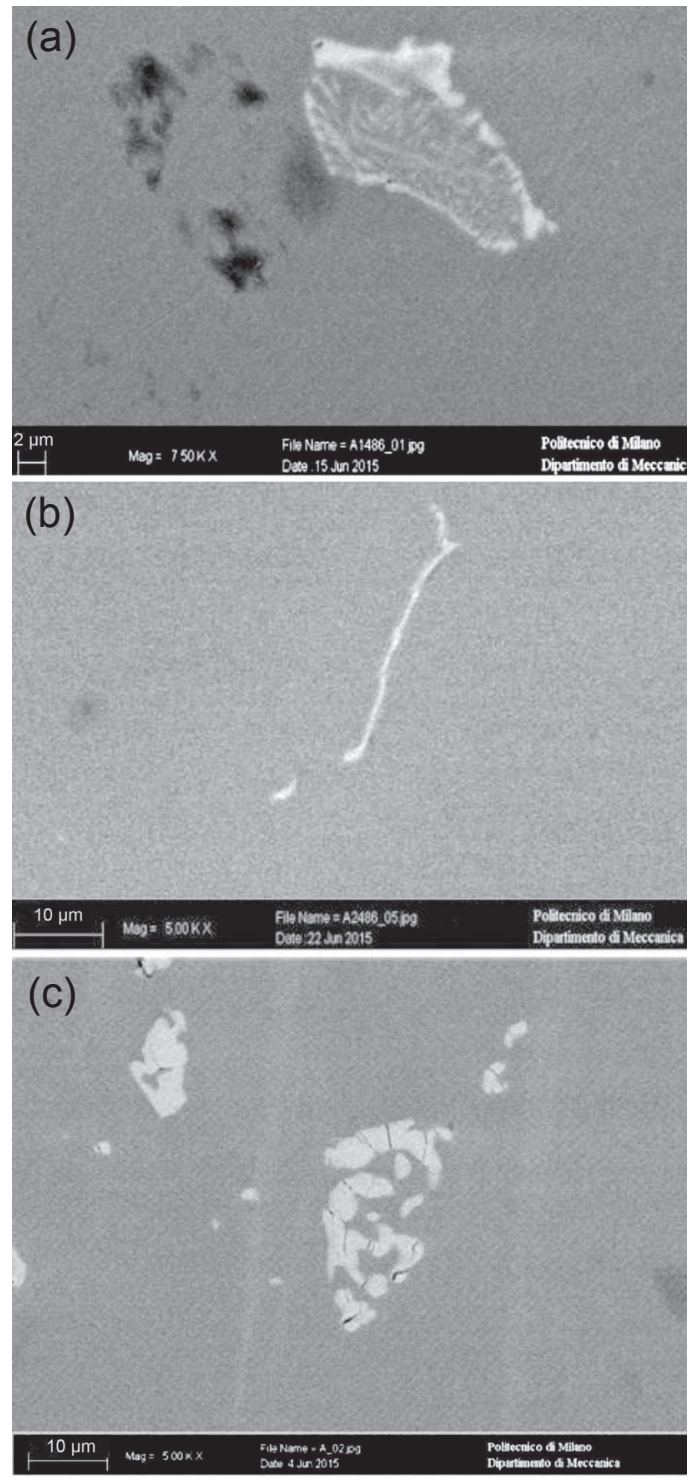

Fig. 7: Two types of $\mathrm{NbC}$ found in alloy 625-1 after cooling at $5 \mathrm{~K} \cdot \mathrm{min}^{-1}$ featuring a lamellar morphology (a) and at $10 \mathrm{~K} \cdot \mathrm{min}^{-1}$ characterised by an elongated shape (Chinese script) (b) and a round shape (c)

Table 5: Chemical compositions (at.\%) from EDX analysis of two NbC morphologies found in alloy IN625-1 after calorimetric tests

\begin{tabular}{|c|c|c|c|c|}
\hline & $\mathrm{Cr}$ & $\mathbf{N i}$ & $\mathrm{Nb}$ & Mo \\
\hline Lamellar & 3.11 & 29.35 & 67.54 & - \\
\hline Chinese script & 7.07 & 17.56 & 73.16 & 2.21 \\
\hline Round shape & 5.12 & 25.64 & 69.24 & - \\
\hline
\end{tabular}



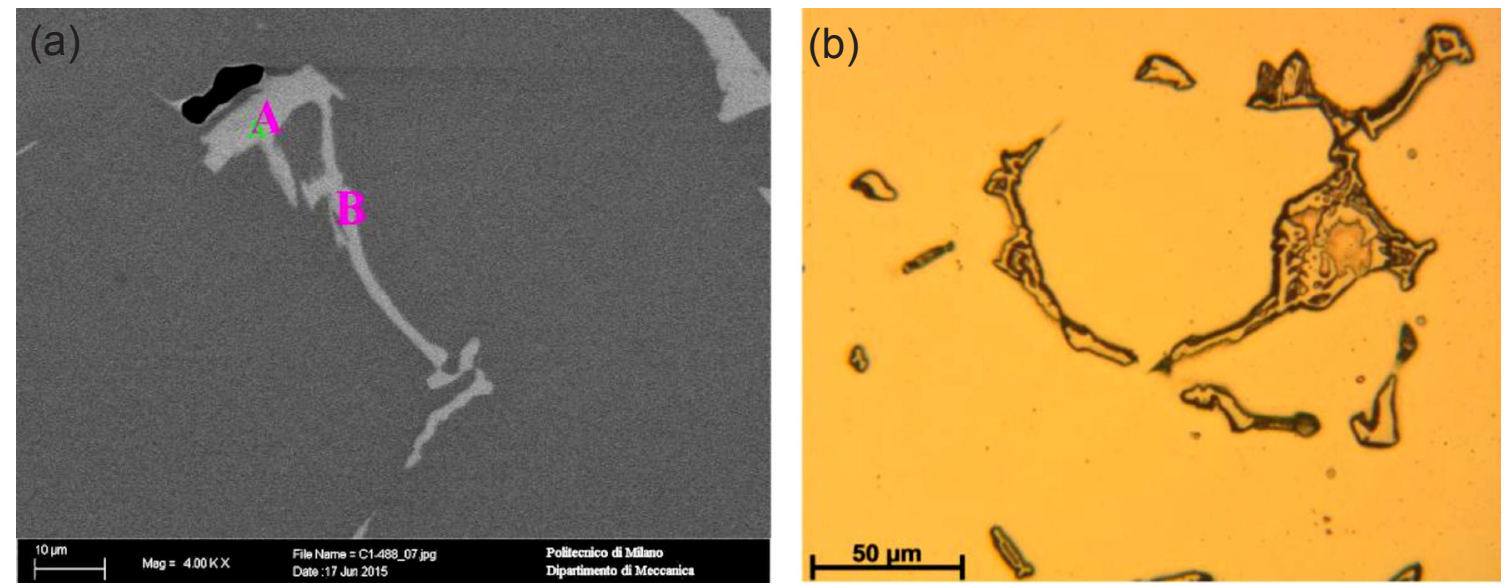

Fig. 8: $\mathrm{NbC}$ characterised by Chinese script morphology found in alloy IN625-3 after a cooling rate of $5 \mathrm{~K} \cdot \mathrm{min}^{-1}$

Table 6: Chemical compositions (at.\%) of complex precipitate in Fig. 8

\begin{tabular}{rcccccccccc} 
& Si & Cr & Mn & Fe & Ni & No & Mo & Phase \\
\hline A & 9.57 & 15.36 & 0.30 & 0.67 & 38.51 & 17.55 & 18.03 & Laves \\
B & - & 7.60 & - & - & 8.62 & 81.59 & 2.19 & NbC \\
\hline
\end{tabular}

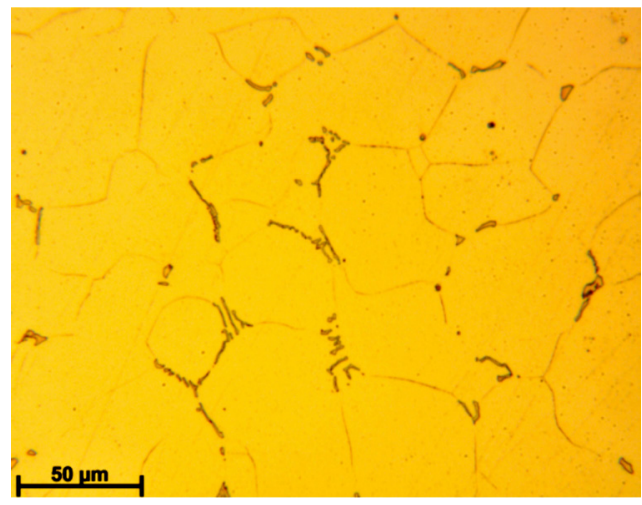

Fig. 9: Microstructural comparison between as-cast condition (a) and cooled at $5 \mathrm{~K} \cdot \mathrm{min}^{-1}$ condition for alloy IN625-4

\subsection{Heat treatments}

Solubilisation treatments (performed at 1,478 K and at 1,548 $\mathrm{K})$ aim at obtaining a decrease in the residual tensions inherited from the solidification process and at taking in solution the alloying elements from the secondary phases. The treatment realized at $1,478 \mathrm{~K}$, usually performed after casting, does not eliminate the undesired secondary phases. This result is consistent with the measured DSC pattern. On the other hand, secondary phase dissolution takes place during the solubilisation treatment performed at 1,548 $\mathrm{K}$, as shown by the DSC analysis. Moreover, the calorimetric analysis suggests higher cooling rates to prevent secondary phase precipitation. For this reason, rapid water quenching was performed after solubilisation.

Assessing the previous statement, only the results related to the $1,548 \mathrm{~K}$ treatment were reported. The effect of this treatment on the alloys IN625-1 and IN625-2 (Fig. 10) implies disconnection of the secondary phase network at the grain boundaries, but complete solubilisation of the secondary phases does not take place. Actually, maintaining at high temperature caused partial re-absorption of the secondary phases, but the subsequent cooling was not enough to completely avoid their large precipitation. The secondary phases accumulated at the grain boundary and some porosities formed, caused by the difference in shrinkage between the precipitates and the matrix (Table 7).

Table 7: Volume fraction (\%) of micro-porosities revealed in different analysed samples after 1,548 $\mathrm{K}$ solubilisation treatment and percentage increment in respect to as-cast condition

\begin{tabular}{|ccc|}
\hline Alloy & $\begin{array}{c}\text { Micro-porosity volume } \\
\text { fraction (\%) }\end{array}$ & Increment (\%) \\
\hline IN625-1 & 0.75 & 30 \\
IN625-2 & 0.45 & 36 \\
IN625-3 & 0.020 & 53 \\
IN625-4 & 0.097 & 12 \\
\hline
\end{tabular}

The presence of the secondary phases in IN625-3 and IN625-4 after the heat treatment at 1,548 $\mathrm{K}$ was higher than in the as-cast condition. Both these alloys (Fig. 11) showed a continuous network of secondary phases formed by the massive precipitation that took place during fast cooling.

Although the carbon concentration in alloy IN625-4 is high, the chemical analysis of the secondary phases (Fig. 12) showed the predominance of Laves phase (Table 8) instead of $\mathrm{NbC}$ precipitates. The elevated cooling rate obtained by water quenching and the contemporary presence of high $\mathrm{Si}$ and $\mathrm{Fe}$ contents in the alloy promoted the formation of the Laves phase, even if a larger quantity of $\mathrm{NbC}$ would be expected ${ }^{[8,15]}$. 

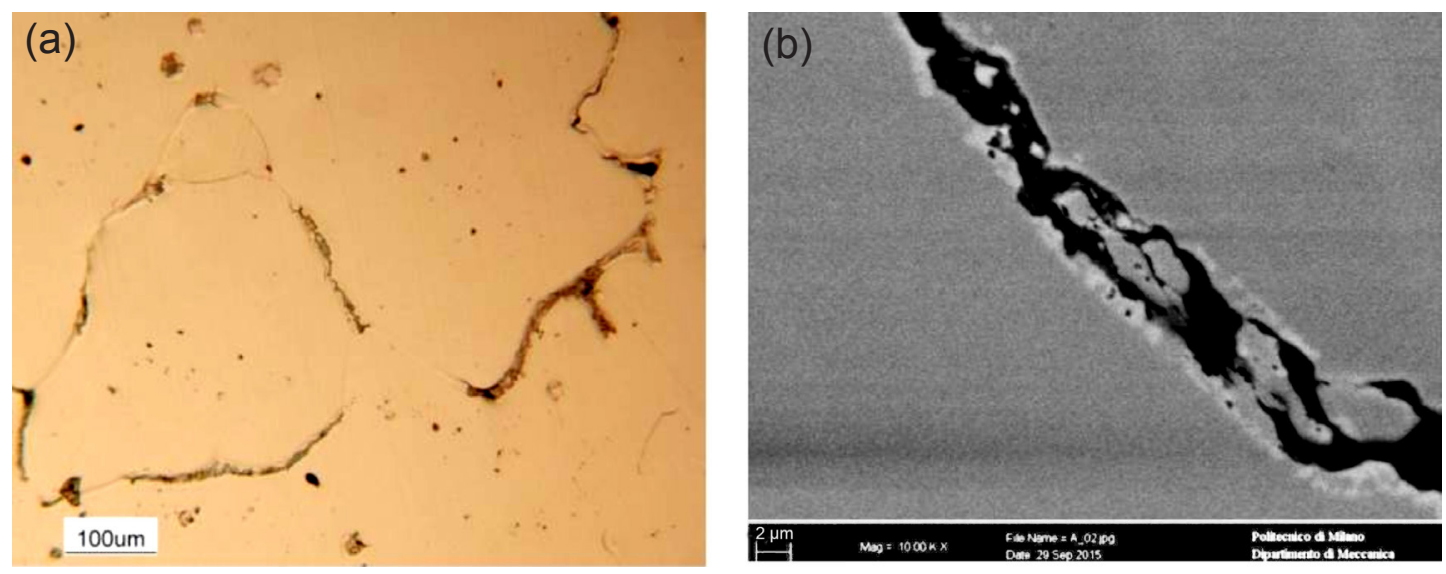

Fig. 10: Accumulation of secondary phases after solution treatment at 1,548 $\mathrm{K}$ that illustrates disconnection of secondary phase network for IN625-1 (a) and IN625-2 (b)
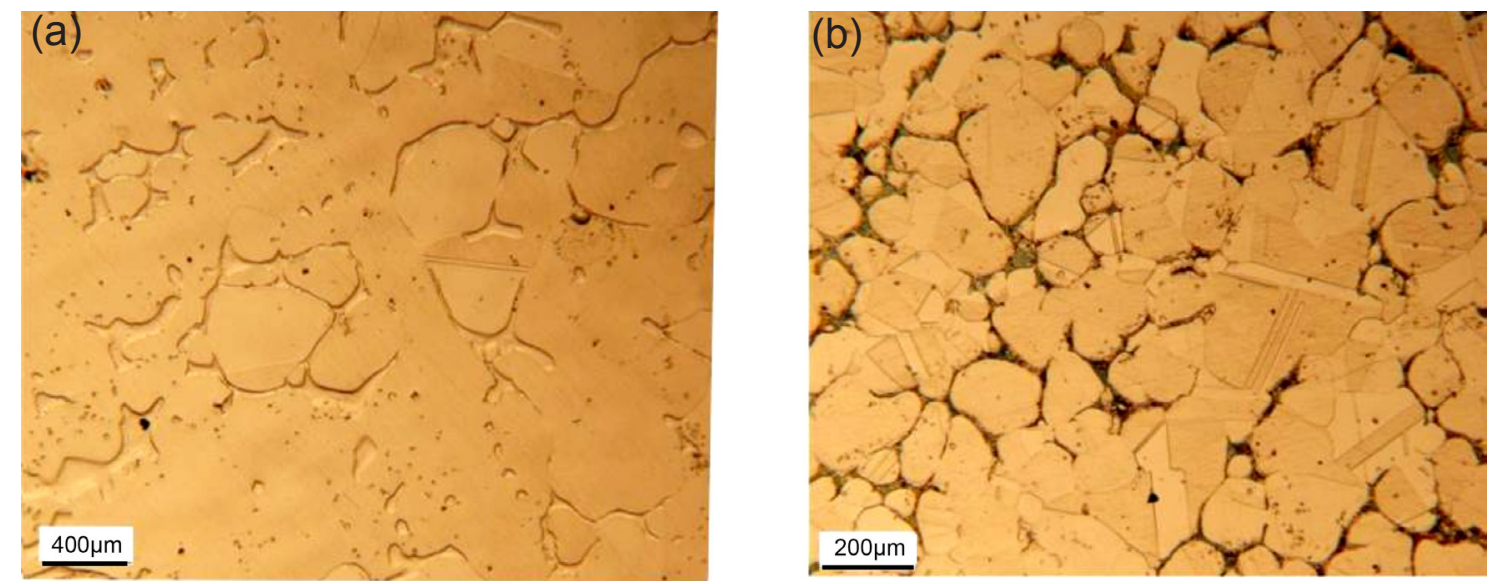

Fig. 11: Microstructures of IN625-3 (a) and IN625-4 (b) after solubilisation heat treatment performed at 1,548 K

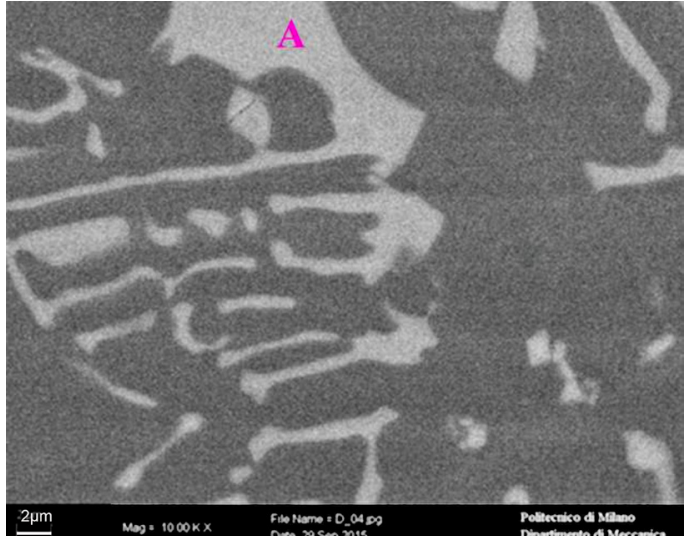

Fig. 12: Presence of Laves phase revealed after solution treatment carried out on alloy IN625-4

Table 8: Chemical compositions (at.\%) of complex precipitate in Fig.12

\begin{tabular}{cccccc}
\hline Si & Cr & Fe & Ni & Nb & Mo \\
\hline 9.45 & 17.84 & 1.23 & 35.70 & 15.25 & 20.54 \\
\hline
\end{tabular}

For all the studied alloys, the amount of the secondary phases after heat treatment at $1,548 \mathrm{~K}$ was greater than that of the ascast condition.

\subsection{Corrosion tests}

Corrosion resistance is one of the most important requirements for these centrifuged spheres. Figure 13 shows the sample weight loss.

Weight loss values are relatively low for all the alloys, because of the high $\mathrm{Cr}$ and Mo content. However, the solubilisation treatment at $1,548 \mathrm{~K}$ reduced the pitting corrosion resistance more than the treatment at $1,478 \mathrm{~K}$ did. This is related to the secondary phase formation during the two different treatments: the higher treatment temperature caused the precipitation of a larger amount of secondary phases, while the lower one, at 1,478 $\mathrm{K}$, gave a precipitation content that was quite similar to that of the as-cast condition.

Weight loss values are relatively low for all the alloys, because of the high $\mathrm{Cr}$ and Mo content. However, the solubilisation treatment at $1,548 \mathrm{~K}$ reduced the pitting corrosion resistance more than the treatment at $1,478 \mathrm{~K}$ did. This is related to the secondary phase formation during the two different treatments: the higher treatment temperature caused the precipitation of a larger amount of secondary phases, while the lower one, at 1,478 $\mathrm{K}$, gave a precipitation content that was quite similar to that of the as-cast condition. 


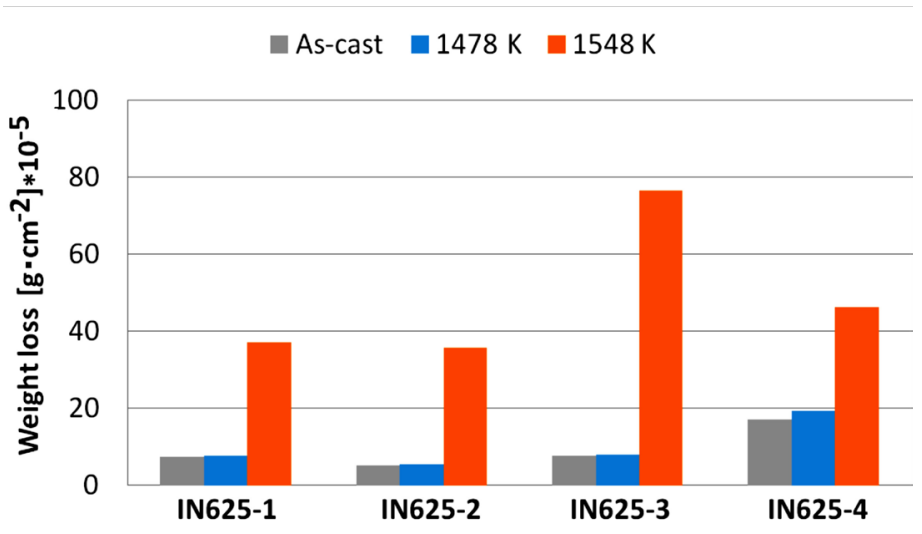

Fig. 13: Weight loss of solution treated samples as a function of alloy chemical compositions and of temperature applied for solution quenching (The experimental trials were performed according to ASTM G48-11)

\section{Discussion}

First of all, the observed micro-porosities were formed as a consequence of the shrinkage difference between the matrix and the secondary phases during both solidification and cooling after thermal treatment. If the secondary phases are characterised by a small size and they form a fine network around the grain boundaries (i.e. Figure 5 for alloy IN625-4), they are less affected by the shrinkage difference occurring with the matrix, and so the formation of micro-porosities is inhibited during solidification and also during the cooling stage of heat treatment

Alloys IN625-3 and IN625-4 feature a higher concentration of the alloying elements ( $\mathrm{Nb}, \mathrm{Mo}, \mathrm{C}$ and $\mathrm{Si}$ ), so the higher imposed constitutional supercooling favours nucleation of the secondary phases. Moreover, the solidification range influences the porosity formation. As reported by Lecomte-Bechers ${ }^{[16]}$, a wide solidification range promotes the porosity formation. Because of the higher solidification range, a greater dendritic length is obtained and, to fulfil the interdentritic spaces, a greater liquid pressure is needed. For this reason, the narrow solidification range measured in IN625-3 and IN625-4 gives reason for the porosity values detected. In addition, it is well known that carbon decreases the molten metal viscosity, so the interdendritic fulfilling is enhanced at the same process pressure: in alloy IN625-4 the higher carbon content allows a reduction in porosity level. Furthermore, as reported by Richards et al, ${ }^{[14]}$ the $\mathrm{C} / \mathrm{Nb}$ ratio influences the solidification path: higher value of this ratio leads to the formation of $\gamma+\mathrm{NbC}$, with no Laves phase. This further reduces the solidification range and, consequently, the porosity level. On the other hand, the lower level of porosity belongs to alloy IN625-3; this can be explained by the Mo concentration. Mo possesses a beneficial effect on porosity because it decreases the liquid and solid density difference and in turn reduces the shrinkage and the porosity formation ${ }^{[16]}$. The effect of Mo on the porosity reduction is further confirmed by the analysis of sample IN625-1 and IN625-2. At the same level of $\mathrm{C}$ and $\mathrm{Nb}$, the only relevant difference is in Mo: alloy IN625-
2 has a higher concentration of these elements and this results in a lower porosity level than alloy IN625-1.

The coexistence of the $\mathrm{NbC}$ and the Laves phase in the same aggregate is an interesting phenomenon that was observed, and is a consequence of the sequence when followed by the precipitation process of the secondary phase: firstly, the formation of the Chinese script $\mathrm{Nb}$-rich carbides takes place and, when there is no more available carbon, the $\mathrm{NbC}$ formation ends and the Laves phase starts its nucleation and growth.

Although alloy IN625-4 is characterised by a higher level of carbon concentration, the chemical analysis of the secondary phases found after heat treatment (Fig. 16) showed there was an important presence of Laves phase instead of $\mathrm{NbC}$ precipitates. The elevated cooling rate obtained from water quenching and the high $\mathrm{Si}$ and Fe concentration in this alloy promoted the formation of the Laves phase, even if a larger volume fraction of $\mathrm{NbC}$ would be expected ${ }^{[8,15]}$. The morphology and distribution of secondary phases are important in the formation of porosity, indeed, by observing all the samples it could be seen that the finer precipitation along the grain boundaries led to low porosity instead of the large aggregate found in the IN625-1 and IN6252 samples. Furthermore, the heat treatment is performed close to the dissolution temperature measured via DSC. This implies the possibility of incipient melting in the Laves Phase, and the consequent formation of micro-porosity caused by different densities between the liquid and solid phases ${ }^{[17]}$. Alloy IN6253 shows the greater increment in percentage porosity (Table 7). This is due to the low $\mathrm{C} / \mathrm{Nb}$ ratio that promotes the Lavers formation during solidification. This eutectic phase tends to melt during the heat treatment at $1,548 \mathrm{~K}$ and consequently forms more porosity than the other alloys. The IN625-4 has the greater $\mathrm{Nb} / \mathrm{C}$ and during the solidification, the $\mathrm{NbC}$ precipitation is favoured. During heat treatment, these precipitates were readsorbed by the matrix but the rapid quenching promotes the Laves formation due to the high cooling rate. This phase forms in the solid state and shrinkage is limited. The other two alloys 
have the same $\mathrm{C} / \mathrm{Nb}$ ratio (greater than alloy IN625-3) and, thus, the same increment in porosity after the heat treatment.

The secondary phases that precipitated at the lowest temperature were poor in $\mathrm{Ni}$ and $\mathrm{Cr}$, which are important elements for assuring the corrosion resistance of the material. Thus, the secondary phases are detrimental to corrosion resistance because they can rob the matrix of elements vital to service performance ${ }^{[18]}$. For this reason, alloys IN625-1 and IN625-2, which are characterised by a lower concentration of alloying elements, present better corrosion resistance than IN625-3 and IN625-4. The presence of secondary phases is more detrimental to local corrosion resistance than the presence of micro-porosities. Laves phases were detected in all samples but the greater amount is in alloy IN625-3 heat treated, because of the lower $\mathrm{C} / \mathrm{Nb}$ ratio. For this reason, the weight loss was greater in this sample. Moreover, the secondary phases are located mainly at the grain boundaries in alloy IN625-3 and IN625-4. As a consequence, these alloys are more susceptible to pitting corrosion and the weight loss is greater.

\section{Conclusions}

The performed experimental trials confirmed the following conclusions:

- The alloy chemical composition influences the solidification range and the secondary phases precipitation path.

- A wide solidification range implies greater interdendritic channels and consequently greater liquid pressure to ensure the fulfilling.

- Alloy IN625-4 has the greater carbon content that widens the solidification range; but on the other hand, it counterbalances this detrimental effect, reducing the liquid viscosity, leading to a better interdendritc channel fulfilling and a reduced porosity.

- Alloy IN625-3 shows the lowest value of $\mathrm{C} / \mathrm{Nb}$ ratio that promotes the Laves phase formation that further widens the solidification range, and consequently increases the porosity. However, the Mo content counterbalances this side effect, reducing the difference between liquid and solid phase density.

- The formation of micro-porosities during heat treatment is governed by the morphology and size of the secondary phases: $\mathrm{Nb}$-rich carbides $(\mathrm{NbC})$ and Laves phase. As their surface to volume ratio increases and their size decreases, they are less affected by the shrinkage difference with the matrix during the cooling stage, so a lower volume fraction of micro-porosities is formed.

- The heat treatment temperature is close to the dissolution temperature, and for this reason, incipient melting in the Laves phases is expected. This leads to the increase in porosity due to the further shrinkage. This effect is more evident in alloy IN625-3 due to the high $\mathrm{C} / \mathrm{Nb}$ ratio.

- The increase in the volume fraction of the secondary phases is detrimental to the local corrosion resistance, as in alloy IN625-3 heat treated. Moreover, the precipitate location (grain boundaries) further decreases the corrosion resistance.

- The chemical composition designed for alloy IN6253 , treated at $1478 \mathrm{~K}$, provides the best combination to assure the absence of micro-porosities and to grant local corrosion resistance.

\section{References}

[1] Beeley P. Foundry Technology. Oxford: Butterworth-Heinemann, 2001: 622-674.

[2] Cumberland J. Centrifugal Casting Techniques. Br Foundrym, 1963,1: 26-47.

[3] Donachie M J, Donachie S J. Superalloys, A Technical Guide. ASM International, 2002: 1-56

[4] Geddes B, Leon H, Huang X. Superalloys, Alloying and Performance. ASM International, 2010: 24-76.

[5] Eiselstein H, Tillack D. The Invention and Definition of Alloy 625. Superalloys 718, 625 Var Deriv, 1991: 1-14.

[6] Cieslak M J. The welding and solidification metallurgy of Alloy 625. Weld J. 1991,70(2): 49-56.

[7] Cieslak M J, Headley T J, Kollie T, et al. A Melting and Solidification Study of Alloy 625. Metall Trans., 1988,19A: 23192331.

[8] Floreen S, Fuchs G, Yang W. the Metallurgy of Alloy-625. Superalloys, 1994: 13-37.

[9] DuPont J, Robino C, Marder A. Modeling solute redistribution and microstructural development in fusion welds of $\mathrm{Nb}$-bearing superalloys. Acta Mater, 1998, 46(13): 4781-4790.

[10] DuPont J N, Marder A R, Notis M R, et al. Solidification of $\mathrm{Nb}$-bearing superalloys: Part II. Pseudoternary solidification surfaces. Metall Mater Trans A, 1998, 29(11): 2797-806.

[11] Chang K-M, Lai H-J, Hwang J-Y. Existence of Laves Phase in Nb-Hardened Superalloys. Superalloys 718, 625, 706 Var Deriv, 1994: 683-694.

[12] Campbell C F. Elements of Metallurgy and Engeneering alloys. ASM International, 2008: 345-347.

[13] Mapelli C, Bosatra M, Morini a, et al. Solidification process, microstructure control and mechanical behaviour in spheres produced in 2205 duplex stainless steel by swirling casting. Int $\mathrm{J}$ Cast Met Res,. 2011, 24(5): 314-326.

[14] Richards N L, Chaturvedi M C. Effect of minor elements on weldability of nickel base superalloys. Int Mater Rev, 2013, 45(3): 109-129.

[15] Hojerslev C, N. T, Hald J. Segregation Effect and Phase Development during Solidification of Alloy 625. Mater Sci Forum, 2006: 506

[16] Lecomte-Beckers J. Study of microporosity formation in nickelbase superalloys. Metall Mater Trans A, 1988, 19(9): 2341-2348.

[17] Lee H-S, Kim D-H, Kim D-S, et al. Microstructural changes by heat treatment for single crystal superalloy exposed at high temperature. J Alloys Compd, 2013, 561: 135-141.

[18] Davis J R. Nickel, Cobalt and their alloys. Materials Park, Ohio: ASM International, 2000: 130 . 